\title{
D-Gulonolactone as a Synthon for L-Noviose: First Preparation of 4-O-Demethyl-L-noviofuranose and Related Derivatives
}

\author{
Marjan Ješelnik, Ivan Leban, Slovenko Polanc, and Marijan Kočevar* \\ Faculty of Chemistry and Chemical Technology, University of Ljubljana, Aškerčeva 5, SI- \\ 1000 Ljubljana, Slovenia \\ marijan.kocevar@uni-lj.si
}

\section{SUPPORTING INFORMATION}

Melting points were determined on a Kofler micro hot stage, and are uncorrected. ${ }^{1} \mathrm{H}$ and ${ }^{13} \mathrm{C}$ NMR (75 MHz) spectra were recorded with the Bruker Avance DPX 300 spectrometer at 302 $\mathrm{K}$, using TMS as an internal standard. The coupling constants $(J)$ are given in Hz. IR spectra were obtained with a Bio-Rad FTS 3000MX spectrophotometer $(\mathrm{KBr}$ pellets for solid products and film on $\mathrm{NaCl}$ plates for liquids). Mass spectra were recorded with a VGAnalytical AutoSpec Q instrument. Elemental analyses (C, H, N) were performed with a Perkin Elmer 2400 CHN Analyzer. Optical rotations were measured in a $1 \mathrm{dm}$ path-length cell on a Perkin-Elmer $241 \mathrm{MC}$ Polarimeter and the concentrations are given in g/100 mL. X-Ray data were collected on Nonius KappaCCD diffractometer. Column chromatography was performed with Silica Gel 60 (Merck, 60-63 $\mu \mathrm{m}$ ). Thin-layer chromatography was carried out on Fluka silica gel TLC-cards. Diethyl ether was dried over sodium and distilled before use. Diazomethane was prepared as described in the literature. ${ }^{18}$ All other reagents were used as received from commercial suppliers (Aldrich, Fluka).

Benzyl 2,3-O-(1-methylethylidene)- $\alpha$-L-lyxofuranosiduronaldehyde [Benzyl (5R)-3,4-O(1-methylethylidene)-L-arabino-pentodialdo-5,2-furanoside] (5).

A solution of sodium periodate $(8.31 \mathrm{~g}, 38.9 \mathrm{mmol})$ in water $(60 \mathrm{~mL})$ was added dropwise over $1 \mathrm{~h}$ to a stirred solution of the diol 4 (11 g, $35.5 \mathrm{mmol})$ in methanol $(180 \mathrm{~mL})$. After 
additional stirring at $\mathrm{rt}$ for $1 \mathrm{~h}$ methanol was evaporated under reduced pressure, water (150 $\mathrm{mL})$ was added and the resulting mixture was extracted with diethyl ether $(6 \times 60 \mathrm{~mL})$. Each extract was washed with saturated aqueous solution of sodium chloride (40 mL), combined phases were dried over $\mathrm{Na}_{2} \mathrm{SO}_{4}$, filtered and evaporated under reduced pressure to give yellow oil 5 (7.22 g, 73\%); $[\alpha]_{\mathrm{D}}+39.6^{\circ}(c 0.53, \mathrm{MeOH}) ; \mathrm{IR} v_{\max } / \mathrm{cm}^{-1} 1737,1495,1456,1373 ;{ }^{1} \mathrm{H}$ $\operatorname{NMR}\left(\mathrm{CDCl}_{3}\right) \delta 1.24(3 \mathrm{H}, \mathrm{s}), 1.31(3 \mathrm{H}, \mathrm{s}), 4.49-4.68(4 \mathrm{H}, \mathrm{m}), 5.21\left(1 \mathrm{H}, \mathrm{dd}, J_{1} 4.3 \mathrm{~Hz}, J_{2} 6\right.$ $\mathrm{Hz}), 5.22(1 \mathrm{H}, \mathrm{s}), 7.34(5 \mathrm{H}, \mathrm{m}), 9.57(1 \mathrm{H}, \mathrm{s}) ;{ }^{13} \mathrm{C} \mathrm{NMR}\left(\mathrm{CDCl}_{3}\right) \delta 25.2,25.4,71.0,79.9,80.3$, 81.4, 101.3, 115.3, 127.8, 127.9, 128.3, 136.8, 199.1; EI-MS m/z 263 [(M-Me) $\left.{ }^{+}, 6\right], 91$ (100). HRMS Calcd for $\mathrm{C}_{14} \mathrm{H}_{15} \mathrm{O}_{5}\left[(\mathrm{M}-\mathrm{Me})^{+}\right]$: 263.0919. Found: 263.0923.

\section{Benzyl 2,3-O-(1-methylethylidene)- $\alpha$-L-lyxofuranosiduronic acid (6).}

A solution of silver nitrate $(4.13 \mathrm{~g}, 24.3 \mathrm{mmol})$ in water $(6 \mathrm{~mL})$ was added to the stirred solution of lyxofuranosiduronaldehide $5(2.03 \mathrm{~g}, 7.30 \mathrm{mmol})$ in ethanol $(50 \mathrm{~mL})$, followed by dropwise addition of a solution of potassium hydroxide $(2.73 \mathrm{~g}, 48.7 \mathrm{mmol})$ in water $(53 \mathrm{~mL})$ (over $1.5 \mathrm{~h}$ ). After $6 \mathrm{~h}$ the suspension was filtered, ethanol was evaporated from the filtrate under reduced pressure and the residual solution was washed with diethyl ether $(3 \times 10 \mathrm{~mL})$. Upon cooling to $0{ }^{\circ} \mathrm{C}$ diethyl ether $(30 \mathrm{~mL})$ was added, the stirred two-phase mixture was carefully acidified to $p \mathrm{H} 4-5$ with hydrochloric acid (1:1). The ethereal phase was separated and the aqueous phase was additionally extracted with diethyl ether $(2 \times 30 \mathrm{~mL})$. The combined extracts were washed with saturated aqueous solution of sodium chloride $(10 \mathrm{~mL})$, dried over $\mathrm{MgSO}_{4}$, filtered and evaporated under reduced pressure to give $\alpha$-Llyxofuranosiduroic acid 6 (1.97 g, 92\%) as a yellow oil, which crystallized as a white solid; mp 97-101 ${ }^{\circ} \mathrm{C}$ (diethyl ether) [Ref. ${ }^{17 b}$ gives for enantiomeric $\alpha$-D-lyxofuranosiduronic acid derivative mp 99-101 $\left.{ }^{\circ} \mathrm{C}\right]$; $[\alpha]_{\mathrm{D}}-60.4^{\circ}$ (c 0.38, MeOH); IR $v_{\max } / \mathrm{cm}^{-1} 1758 \mathrm{br}, 1558 \mathrm{br}, 1495$; ${ }^{1} \mathrm{H}$ NMR $\left(\mathrm{CDCl}_{3}\right) \delta 1.30(3 \mathrm{H}, \mathrm{s}), 1.45(3 \mathrm{H}, \mathrm{s}), 4.52(1 \mathrm{H}, \mathrm{d}, J 12.0 \mathrm{~Hz}), 4.70(3 \mathrm{H}, \mathrm{m}), 5.06$ $\left(1 \mathrm{H}, \mathrm{dd}, J_{1} 4.3 \mathrm{~Hz}, J_{2} 5.8 \mathrm{~Hz}\right), 5.28(1 \mathrm{H}, \mathrm{s}), 7.32(5 \mathrm{H}, \mathrm{m}), 8.76(1 \mathrm{H}, \mathrm{br} \mathrm{s}) ;{ }^{13} \mathrm{C} \mathrm{NMR}\left(\mathrm{CDCl}_{3}\right) \delta$ 25.3, 26.3, 69.8, 79.9, 80.9, 84.6, 106.1, 113.9, 128.4, 128.5, 128.9, 137.2, 172.1; EI-MS m/z $\left.295(\mathrm{MH})^{+}, 2\right], 279$ (M-Me), 91 (100). HRMS Calcd for $\mathrm{C}_{15} \mathrm{H}_{19} \mathrm{O}_{6}\left(\mathrm{MH}^{+}\right)$: 295.1181. Found: 295.1193.

\section{1-O-Benzyl methyl 2,3-O-(1-methylethylidene)- $\alpha$-L-lyxofuranosiduronate (7).}

Lyxofuranosiduronic acid 6 (1.65 g, $5.6 \mathrm{mmol})$ was dissolved in diethyl ether $(20 \mathrm{~mL})$ and treated at $0{ }^{\circ} \mathrm{C}$ with etheral solution of diazomethane until nitrogen evolution was completed. 
After evaporation under reduced pressure the compound $7(1.71 \mathrm{~g}, 99 \%)$ was obtained as colorless oil, which later crystallized as a white solid; mp $50-53{ }^{\circ} \mathrm{C}$ (diethyl ether); $[\alpha]_{\mathrm{D}}$ $-43.9^{\circ}\left(c\right.$ 1.05, $\left.\mathrm{CHCl}_{3}\right)$ [Ref. ${ }^{17 \mathrm{~b}}$ gives for enantiomeric $\alpha$-D-lyxofuranosiduronic acid methyl ester $\left.[\alpha]_{\mathrm{D}}+46.4^{\circ}\right]$; IR $v_{\max } / \mathrm{cm}^{-1} 1735,1496,1466,1453,1437 ;{ }^{1} \mathrm{H}$ NMR (DMSO- $\left.d_{6}\right) \delta 1.24$ $(3 \mathrm{H}, \mathrm{s}), 1.30(3 \mathrm{H}, \mathrm{s}), 3.68(3 \mathrm{H}, \mathrm{s}), 4.48(1 \mathrm{H}, \mathrm{d}, J 12.1 \mathrm{~Hz}), 4.63(3 \mathrm{H}, \mathrm{m}), 5.06\left(1 \mathrm{H}, \mathrm{dd}, J_{1} 4.5\right.$ $\left.\mathrm{Hz}, J_{2} 6.0 \mathrm{~Hz}\right), 5.18(1 \mathrm{H}, \mathrm{s}), 7.30(5 \mathrm{H}, \mathrm{m}) ;{ }^{13} \mathrm{C} \mathrm{NMR}\left(\mathrm{CDCl}_{3}\right) \delta 24.9,25.8,52.1,69.2,79.6$, 80.5, 84.0, 105.6, 113.3, 127.9, 128.0, 128.4, 136.8, 167.8; EI-MS $m / z 293$ [(M-Me $\left.)^{+}, 12\right], 91$ (100\%). Anal. Calcd for $\mathrm{C}_{16} \mathrm{H}_{20} \mathrm{O}_{6} \times 0.5$ EtOAc: C, 61.35; H, 6.86. Found: C, 61.14; H, 6.82.

\section{Benzyl 5,5-di- $C$-methyl-2,3-O-(1-methylethylidene)- $\alpha$-L-lyxofuranoside [Benzyl 6-deoxy-} 5-C-methyl-2,3-O-(1-methylethylidene)- $\alpha$-L-lyxo-hexofuranoside ] (8a).

Iodine (ca. $15 \mathrm{mg}, 0.06 \mathrm{mmol}$ ) was added to the stirred suspension of Grignard-grade magnesium (300 mg, $12.3 \mathrm{mmol}$ ) in $20 \mathrm{~mL}$ of diethyl ether, followed by a dropwise addition of a solution of freshly distilled methyl iodide $(1.17 \mathrm{~mL}, 18.8 \mathrm{mmol})$ in diethyl ether $(3 \mathrm{~mL})$. Gentle boiling of the reaction mixture controlled the addition. The reaction mixture was heated for additional $30 \mathrm{~min}$ at the reflux temperature. The solution of a methyl ester 7 (1.59 $\mathrm{g}, 5.16 \mathrm{mmol})$ in diethyl ether $(5 \mathrm{~mL})$ was added dropwise to the solution of methylmagnesium iodide in diethyl ether and the reaction mixture was heated for $2 \mathrm{~h}$, then it was allowed to cool to $\mathrm{rt}$ and poured onto ice-cold $17 \%$ solution of ammonium chloride $(50 \mathrm{~mL})$. Layers were separated and the aqueous layer was extracted with diethyl ether $(2 \times 20 \mathrm{~mL})$, combined organic layers were dried over $\mathrm{Na}_{2} \mathrm{SO}_{4}$, filtered and evaporated under reduced pressure to give $8 \mathbf{a}(1.51 \mathrm{~g}, 95 \%)$ as yellowish oil, which later crystallized as a white solid; mp 39-40 ${ }^{\circ} \mathrm{C}$ (diethyl ether); $[\alpha]_{\mathrm{D}}-83.3^{\circ}$ (c 0.56, MeOH); IR $v_{\max } / \mathrm{cm}^{-1} 1497,1455,1401$, 1383, 1375, 1364; ${ }^{1} \mathrm{H}$ NMR (DMSO-d $) \delta 1.20(3 \mathrm{H}, \mathrm{s}), 1.24(6 \mathrm{H}, \mathrm{s}), 1.36(3 \mathrm{H}, \mathrm{s}), 3.73(1 \mathrm{H}, \mathrm{d}$, $J 3.4 \mathrm{~Hz}), 4.29(1 \mathrm{H}, \mathrm{s}), 4.47(1 \mathrm{H}, \mathrm{d}, J 12 \mathrm{~Hz}), 4.56(1 \mathrm{H}, \mathrm{d}, J 6.0 \mathrm{~Hz}), 4.61(1 \mathrm{H}, \mathrm{d}, J 12 \mathrm{~Hz})$, $4.77\left(1 \mathrm{H}, \mathrm{dd}, J_{1} 3.4 \mathrm{~Hz}, J_{2} 6.0 \mathrm{~Hz}\right), 5.05(1 \mathrm{H}, \mathrm{s}), 7.29(5 \mathrm{H}, \mathrm{m}) ;{ }^{13} \mathrm{C} \mathrm{NMR}\left(\mathrm{CDCl}_{3}\right) \delta 24.5$, 26.1, 27.62, 27.65, 69.5, 71.2, 81.1, 83.5, 85.7, 105.3, 113.1, 128.2, 128.4, 128.8, 137.7; FAB-MS $m / z 309\left(\mathrm{MH}^{+}\right)$. Anal. Calcd for $\mathrm{C}_{17} \mathrm{H}_{24} \mathrm{O}_{5}$ : C, 66.21; H, 7.84. Found: $\mathrm{C}, 66.34 ; \mathrm{H}$, 7.56 .

\section{Benzyl 2,3-O-(1-methylethylidene)-5,5-di- $C$-propyl- $\alpha$-L-lyxofuranoside (8b).}

To the stirred solution of $2 \mathrm{M}$ propylmagnesium chloride in diethyl ether $(16.2 \mathrm{~mL}, 32.4$ mmol) and additional diethyl ether $(20 \mathrm{~mL})$ a solution of methyl ester 7 (4 g, $13.0 \mathrm{mmol})$ in 
diethyl ether $(10 \mathrm{~mL})$ was added dropwise to keep the mixture gently boiling. The reaction mixture was heated for additional $2 \mathrm{~h}$ at the reflux temperature, and then it was allowed to cool to $\mathrm{rt}$ and poured onto ice-cold $17 \%$ solution of ammonium chloride (130 mL). Layers were separated and the aqueous layer was extracted with diethyl ether $(2 \times 20 \mathrm{~mL})$, combined organic layers were dried over $\mathrm{Na}_{2} \mathrm{SO}_{4}$, filtered and evaporated under reduced pressure. The crude product was purified by column chromatography (ethyl acetate-petroleum ether 1:20) to give 12b (2.30 g, 49\%) as a yellowish oil, which later crystallized as a white solid; mp $50-51.5{ }^{\circ} \mathrm{C}$ (diethyl ether); $[\alpha]_{\mathrm{D}}-71.6^{\circ}$ (c $\left.0.75, \mathrm{MeOH}\right) ; \mathrm{IR} v_{\max } / \mathrm{cm}^{-1} 1456,1424,1377$, 1274, 1213, 1084, 1030; ${ }^{1} \mathrm{H}$ NMR $\left(\mathrm{CDCl}_{3}\right) \delta 0.95(6 \mathrm{H}, \mathrm{m}), 1.24-1.72(8 \mathrm{H}, \mathrm{m}), 1.31(3 \mathrm{H}, \mathrm{s})$, $1.50(3 \mathrm{H}, \mathrm{s}), 3.60(1 \mathrm{H}, \mathrm{s}), 3.83(1 \mathrm{H}, \mathrm{d}, J 3.2 \mathrm{~Hz}), 4.50,(1 \mathrm{H}, \mathrm{d}, J 11.7 \mathrm{~Hz}), 4.64(1 \mathrm{H}, \mathrm{d}, J 5.8$ Hz), $4.65(1 \mathrm{H}, \mathrm{d}, J 11.7 \mathrm{~Hz}), 4.86\left(1 \mathrm{H}, \mathrm{dd}, J_{1} 3.2 \mathrm{~Hz}, J_{2} 5.8 \mathrm{~Hz}\right), 5.18(1 \mathrm{H}, \mathrm{s}), 7.32(5 \mathrm{H}, \mathrm{m})$; ${ }^{1} \mathrm{H}$ NMR (DMSO- $\left.d_{6}\right) \delta 0.95(6 \mathrm{H}, \mathrm{m}), 1.20-1.70(8 \mathrm{H}, \mathrm{m}), 1.30(3 \mathrm{H}, \mathrm{s}), 1.49(3 \mathrm{H}, \mathrm{s}), 3.60(1 \mathrm{H}$, br s), $3.83(1 \mathrm{H}, \mathrm{d}, J 3.4 \mathrm{~Hz}), 4.49$, (1H, d, J $11.7 \mathrm{~Hz}), 4.64(1 \mathrm{H}, \mathrm{d}, J 5.9 \mathrm{~Hz}), 4.65(1 \mathrm{H}, \mathrm{d}, J$ $11.7 \mathrm{~Hz}), 4.85\left(1 \mathrm{H}, \mathrm{dd}, J_{1} 3.4 \mathrm{~Hz}, J_{2} 5.9 \mathrm{~Hz}\right), 5.17(1 \mathrm{H}, \mathrm{s}), 7.31(5 \mathrm{H}, \mathrm{m}) ;{ }^{13} \mathrm{C} \mathrm{NMR}\left(\mathrm{CDCl}_{3}\right) \delta$ 14.7, 14.8, 16.6 (two signals), 24.2, 25.7, 37.8, 39.0, 69.1, 74.9, 80.6, 80.8, 85.2, 104.6, 112.7, 127.8, 128.1, 128.4, 137.3; FAB-MS $m / z 365\left(\mathrm{MH}^{+}\right)$. HRMS Calcd for $\mathrm{C}_{20} \mathrm{H}_{29} \mathrm{O}_{5}\left[(\mathrm{M}-\mathrm{Me})^{+}\right]$: 349.2015. Found: 349.2030 .

\section{Benzyl 2,3-O-(1-methylethylidene)-5,5-di- $C$-phenyl- $\alpha$-L-lyxofuranoside (8c).}

To the stirred solution of $3 \mathrm{M}$ phenylmagnesium chloride in diethyl ether $(5.4 \mathrm{~mL}, 16.2$ mmol) and additional diethyl ether $(15 \mathrm{~mL})$ a solution of methyl ester 7 (2 g, $6.5 \mathrm{mmol})$ in diethyl ether $(10 \mathrm{~mL})$ was added dropwise as fast that the mixture was gently boiling; then the reaction mixture was heated for additional $2 \mathrm{~h}$ at the reflux temperature. Upon cooling to $\mathrm{rt}$ it was poured onto ice-cold $17 \%$ solution of ammonium chloride $(60 \mathrm{~mL})$. Layers were separated and the aqueous layer was extracted with diethyl ether $(3 \times 20 \mathrm{~mL})$. Combined extracts were dried over $\mathrm{Na}_{2} \mathrm{SO}_{4}$, filtered and evaporated under reduced pressure. The crude product was purified by column chromatography (ethyl acetate-petroleum ether 1:20) to give yellow oil 8c (2.28 g, 82\%), which crystallized as a white solid; mp 95.2-96.4 ${ }^{\circ} \mathrm{C}$ (diethyl ether); $[\alpha]_{\mathrm{D}}-44.1^{\circ}(c 0.81, \mathrm{MeOH}) ; \mathrm{IR} v_{\max } / \mathrm{cm}^{-1} 1493,1448,1381,1273,1212 ;{ }^{1} \mathrm{H} \mathrm{NMR}$ $\left(\mathrm{DMSO}-d_{6}\right) \delta 1.16(3 \mathrm{H}, \mathrm{s}), 1.33(3 \mathrm{H}, \mathrm{s}), 4.42(1 \mathrm{H}, \mathrm{d}, J 11.7 \mathrm{~Hz}), 4.53(1 \mathrm{H}, \mathrm{d}, J 11.7 \mathrm{~Hz}), 4.64$ $(1 \mathrm{H}, \mathrm{d}, J 5.9 \mathrm{~Hz}), 4.82\left(1 \mathrm{H}, \mathrm{dd}, J_{1} 3.3 \mathrm{~Hz}, J_{2} 5.9 \mathrm{~Hz}\right), 5.03(1 \mathrm{H}, \mathrm{d}, J 3.3 \mathrm{~Hz}), 5.12(1 \mathrm{H}, \mathrm{s}), 5.15$ $(1 \mathrm{H}, \mathrm{s}), 7.12-7.67(15 \mathrm{H}, \mathrm{m}) ;{ }^{13} \mathrm{C} \mathrm{NMR}\left(\mathrm{CDCl}_{3}\right) \delta$ 24.7, 26.3, 69.9, 78.6, 81.0, 81.6, 85.6, $105.9,113.6,125.9,126.1,127.2$, 127.4, 128.4, 128.5, 128.6, 128.7, 129.0, 137.5, 145.4, 
145.9; FAB-MS $m / z 415$ [(MH- $\left.\left.\mathrm{H}_{2} \mathrm{O}\right)^{+}\right]$. Anal. Calcd for $\mathrm{C}_{27} \mathrm{H}_{28} \mathrm{O}_{5}: \mathrm{C}, 74.98 ; \mathrm{H}, 6.53$. Found: C, 74.88; H. 6.69.

2,3-O-(1-Methylethylidene)-5,5-di- $C$-methyl- $\alpha$-L-lyxofuranose [6-Deoxy-5- $C$-methyl-2,3$O$-(1-methylethylidene)- $\alpha$-L-lyxo-hexofuranose] (9a).

To the solution of $8 \mathbf{a}(1.69 \mathrm{~g}, 5.49 \mathrm{mmol})$ in diethyl ether $(50 \mathrm{~mL}) 10 \% \mathrm{Pd} / \mathrm{C}(200 \mathrm{mg})$ was added, then it was hydrogenated at 4.1 bar (60 psi) for $5 \mathrm{~h}$. The resulting suspension was filtered through a celite pad and evaporated under diminished pressure to give 9a $(1.18 \mathrm{~g}$, 99\%) as a white solid; mp $73-77^{\circ} \mathrm{C}$ (ethanol); $[\alpha]_{\mathrm{D}}-31.0^{\circ}(c 0.55, \mathrm{MeOH}) ; \mathrm{IR} v_{\max } / \mathrm{cm}^{-1}$ 1462, 1376, 1273, 1244, 1211; ${ }^{1} \mathrm{H}$ NMR (DMSO-d $d_{6} \delta 1.19(3 \mathrm{H}, \mathrm{s}), 1.21(3 \mathrm{H}, \mathrm{s}), 1.23(3 \mathrm{H}, \mathrm{s})$, $1.35(3 \mathrm{H}, \mathrm{s}), 3.79(1 \mathrm{H}, \mathrm{d}, J 3.4 \mathrm{~Hz}), 4.15(1 \mathrm{H}, \mathrm{s}), 4.42(1 \mathrm{H}, \mathrm{d}, J 5.8 \mathrm{~Hz}), 4.73\left(1 \mathrm{H}, \mathrm{dd}, J_{1} 3.4\right.$ $\left.\mathrm{Hz}, J_{2} 5.8 \mathrm{~Hz}\right), 5.15(1 \mathrm{H}, \mathrm{d}, J 4.1 \mathrm{~Hz}), 6.28(1 \mathrm{H}, \mathrm{d}, J 4.1 \mathrm{~Hz}) ;{ }^{13} \mathrm{C} \mathrm{NMR}\left(\mathrm{CDCl}_{3}\right) \delta 24.1,25.7$, 27.2, 27.3, 71.1, 80.9, 83.3, 85.7, 100.6, 112.8; EI-MS m/z 203 [(M-Me) $\left.{ }^{+}, 18\right], 59$ (100). HRMS Calcd for $\mathrm{C}_{9} \mathrm{H}_{15} \mathrm{O}_{5}\left[(\mathrm{M}-\mathrm{Me})^{+}\right]$: 203.0919. Found: 203.0923.

\section{2,3-O-(1-Methylethylidene)-5,5-di- $C$-propyl- $\alpha$-L-lyxofuranose (9b).}

To the solution of $\mathbf{8 b}(885 \mathrm{mg}, 2.4 \mathrm{mmol})$ in diethyl ether $(50 \mathrm{~mL}) 10 \% \mathrm{Pd} / \mathrm{C}(180 \mathrm{mg})$ was added, then it was hydrogenated at 4.1 bar (60 psi) for $4.5 \mathrm{~h}$. The resulting suspension was filtered through a celite pad and evaporated under diminished pressure to give $\mathbf{9 b}(510 \mathrm{mg}$, $76 \%$ ) as a yellowish oil, which later crystallized as a white solid; mp $66.5-69{ }^{\circ} \mathrm{C}$ (diethyl ether); $[\alpha]_{\mathrm{D}}-22.4^{\circ}(c 0.37, \mathrm{MeOH}) ; \mathrm{IR} v_{\max } / \mathrm{cm}^{-1} 1459,1425,1377,1212 ;{ }^{1} \mathrm{H} \mathrm{NMR}\left(\mathrm{CDCl}_{3}\right)$ $\delta 0.93(6 \mathrm{H}, \mathrm{m}), 1.27-1.64(8 \mathrm{H}, \mathrm{m}), 1.32(3 \mathrm{H}, \mathrm{s}), 1.50(3 \mathrm{H}, \mathrm{s}), 3.73(1 \mathrm{H}, \mathrm{br} \mathrm{s}), 3.98(1 \mathrm{H}, \mathrm{d}, J$ $3.4 \mathrm{~Hz}), 4.59(1 \mathrm{H}, \mathrm{d}, J 5.8 \mathrm{~Hz}), 4.87\left(1 \mathrm{H}, \mathrm{dd}, J_{1} 3.4 \mathrm{~Hz}, J_{2} 5.8 \mathrm{~Hz}\right), 5.45(1 \mathrm{H}, \mathrm{s}) ;{ }^{1} \mathrm{H}$ NMR (DMSO- $\left.d_{6}\right) ; \delta 0.86(6 \mathrm{H}, \mathrm{m}), 1.20-1.55(8 \mathrm{H}, \mathrm{m}), 1.25(3 \mathrm{H}, \mathrm{s}), 1.37(3 \mathrm{H}, \mathrm{s}), 3.71(1 \mathrm{H}, \mathrm{s}), 3.81$ $(1 \mathrm{H}, \mathrm{d}, J 3.4 \mathrm{~Hz}), 4.44(1 \mathrm{H}, \mathrm{d}, J 5.8 \mathrm{~Hz}), 4.87\left(1 \mathrm{H}, \mathrm{dd}, J_{1} 3.4 \mathrm{~Hz}, J_{2} 5.8 \mathrm{~Hz}\right), 5.14(1 \mathrm{H}, \mathrm{d}, J 4.1$ $\mathrm{Hz}), 6.30(1 \mathrm{H}, \mathrm{d}, 4.1 \mathrm{~Hz}) ;{ }^{13} \mathrm{C} \mathrm{NMR}\left(\mathrm{CDCl}_{3}\right) \delta 14.5,14.7,16.4,16.5,24.1,25.7,37.6,38.7$, 75.2, 80.5, 80.9, 85.7, 100.0, 112.6; FAB-MS m/z $275\left(\mathrm{MH}^{+}\right)$. Anal. Calcd for $\mathrm{C}_{14} \mathrm{H}_{26} \mathrm{O}_{5}: \mathrm{C}_{\text {, }}$ 61.29; H, 9.55. Found: C, 61.02; H, 9.89.

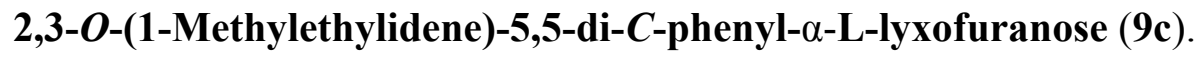

To the solution of $8 \mathrm{c}(1.62 \mathrm{~g}, 3.7 \mathrm{mmol})$ in diethyl ether $(50 \mathrm{~mL}) 10 \% \mathrm{Pd} / \mathrm{C}(300 \mathrm{mg})$ was added, then it was hydrogenated at 4.1 bar (60 psi) for $12 \mathrm{~h}$. The resulting suspension was filtered through a celite pad, evaporated under reduced pressure to give $9 \mathbf{c}(600 \mathrm{mg}, 47 \%)$ as a 
yellowish oil, which crystallized as a white solid; mp $138-140{ }^{\circ} \mathrm{C}$ (diethyl ether); IR $v_{\max } / \mathrm{cm}^{-1} 1599,1495,1450,1378$ br, 1337, 1318, 1276; ${ }^{1} \mathrm{H}$ NMR $\left(\mathrm{CDCl}_{3}\right) \delta 1.18(3 \mathrm{H}, \mathrm{s})$, 1.53, (3H, s), $3.15\left(1 \mathrm{H}\right.$, br s), $4.55(1 \mathrm{H}, \mathrm{d}, J 5.8 \mathrm{~Hz}), 4.66\left(1 \mathrm{H}, \mathrm{dd}, J_{1} 3.0 \mathrm{~Hz}, J_{2} 5.8 \mathrm{~Hz}\right), 5.07$ $(1 \mathrm{H}, \mathrm{d}, J 3.0 \mathrm{~Hz}), 5.13(1 \mathrm{H}, \mathrm{s}), 5.42(1 \mathrm{H}, \mathrm{s}), 7.22(6 \mathrm{H}, \mathrm{m}), 7.55(4 \mathrm{H}, \mathrm{m}) ;{ }^{1} \mathrm{H}$ NMR (DMSO-d $)$ $\delta 1.15(3 \mathrm{H}, \mathrm{s}), 1.33(3 \mathrm{H}, \mathrm{s}), 4.49(1 \mathrm{H}, \mathrm{d}, J 5.8 \mathrm{~Hz}), 4.78\left(1 \mathrm{H}, \mathrm{dd}, J_{1} 3.4 \mathrm{~Hz}, J_{2} 5.8 \mathrm{~Hz}\right), 5.06$ $(1 \mathrm{H}, \mathrm{d}, J 3.4 \mathrm{~Hz}), 5.08(1 \mathrm{H}, \mathrm{s}), 5.20(1 \mathrm{H}, \mathrm{d}, \mathrm{J} 4.3 \mathrm{~Hz}), 6.56(1 \mathrm{H}, \mathrm{d}, J 4.3 \mathrm{~Hz}), 7.25(6 \mathrm{H}, \mathrm{m})$, $7.48(2 \mathrm{H}, \mathrm{m}), 7.57(2 \mathrm{H}, \mathrm{m}) ;{ }^{13} \mathrm{C} \mathrm{NMR}\left(\mathrm{CDCl}_{3}\right) \delta 24.1,25.7,78.1,80.6,81.2,85.4,100.8$, $113.0,125.4,125.8,126.7,126.9,128.0,128.2,144.9,145.6 ;$ FAB-MS m/z 325 [(MH$\left.\mathrm{H}_{2} \mathrm{O}\right)^{+}$]. Anal. Calcd for $\mathrm{C}_{20} \mathrm{H}_{22} \mathrm{O}_{5}$ : C, 70.16; H, 6.48. Found: C, 70.35; H, 6.69.

\section{4-O-Methyl-5,5-di- $C$-methyl-2,3-O-(1-methylethylidene)-L-lyxopyranose $\quad$ [6-Deoxy-5- $C$ - methyl-4-O-methyl-2,3-O-(1-methylethylidene)-L-lyxo-hexopyranose] (10a).}

To the solution of lyxofuranose 9a $(1.05 \mathrm{~g}, 4.8 \mathrm{mmol})$ in a mixture of toluene $(14 \mathrm{~mL})$ and methylene chloride (6 mL) tetrabutylammonium bromide (213 mg, $0.66 \mathrm{mmol}), 20 \%$ solution of sodium hydroxide $(10 \mathrm{~mL})$ and dimethyl sulfate $(1.3 \mathrm{~mL}, 13.6 \mathrm{mmol})$ were added. The reaction was carried out in the two-phase mixture under phase-transfer conditions for $22 \mathrm{~h}$ at rt. Layers were separated, the aqueous layer was diluted with water $(30 \mathrm{~mL})$ and extracted with methylene chloride $(3 \times 20 \mathrm{~mL})$. Combined organic extracts were dried over $\mathrm{Na}_{2} \mathrm{SO}_{4}$, filtered and evaporated under reduced pressure. Yellow oil was purified by column chromatography (chloroform-petroleum ether $1: 2)$ to give $\mathbf{1 0 a}(0.68 \mathrm{~g}, 61 \%)$ as a yellow oil; $[\alpha]_{\mathrm{D}}-78.7^{\circ}(c 1.56, \mathrm{MeOH})$ lit. $^{12}[\alpha]_{\mathrm{D}}-79.1^{\circ}$ (c 1.58, MeOH); IR $v_{\max } / \mathrm{cm}^{-1} 1727,1464$, 1375, 1271. ${ }^{13} \mathrm{C}$ NMR (DMSO- $d_{6}$ ): $\delta 24.5,26.1,27.64,27.65,54.8,71.2,81.0,83.3,85.6$, 106.9, 113.1; FAB-MS m/z $233\left(\mathrm{MH}^{+}\right) ;{ }^{1} \mathrm{H}$ NMR $\left(\mathrm{CDCl}_{3}\right): \delta 1.32(3 \mathrm{H}, \mathrm{s}), 1.35(3 \mathrm{H}, \mathrm{s}), 1.38$ $(3 \mathrm{H}, \mathrm{s}), 1.51(3 \mathrm{H}, \mathrm{s}), 3.33(3 \mathrm{H}, \mathrm{s}), 3.54(1 \mathrm{H}, \mathrm{s}), 3.69(1 \mathrm{H}, \mathrm{d}, J 3.4 \mathrm{~Hz}), 4.57(1 \mathrm{H}, \mathrm{d}, J 5.8 \mathrm{~Hz})$, $4.84\left(1 \mathrm{H}, \mathrm{dd}, J_{1} 3.4 \mathrm{~Hz}, J_{2} 5.8 \mathrm{~Hz}\right), 5.00(1 \mathrm{H}, \mathrm{s})$.

\section{4-O-Methyl-2,3-O-(1-methylethylidene)-5,5-di-C-propyl-L-lyxopyranose (10b).}

Lyxofuranose 9b (934 mg, $3.4 \mathrm{mmol}$ ) was dissolved in the mixture of methylene chloride (4 $\mathrm{mL})$ and toluene $(10 \mathrm{~mL})$. To this solution tetrabutylammonium bromide $(150 \mathrm{mg}, 0.46$ mmol), $20 \%$ solution of sodium hydroxide $(7 \mathrm{~mL})$ and dimethyl sulfate $(0.92 \mathrm{~mL}, 9.6 \mathrm{mmol})$ were added. The reaction was carried out in a two-phase mixture under phase-transfer conditions for $12 \mathrm{~h}$ at rt. Layers were separated; the aqueous layer was diluted with additional water $(30 \mathrm{~mL})$ and extracted with methylene chloride $(3 \times 15 \mathrm{~mL})$. Combined organic extracts 
were dried over $\mathrm{Na}_{2} \mathrm{SO}_{4}$, filtered and evaporated under reduced pressure. Yellow oil obtained was purified by column chromatography (gradient ethyl acetate-petroleum ether 1:20 to 1:10), to give product $10 \mathrm{~b}(770 \mathrm{mg}, 78 \%)$ as a yellowish oil; $[\alpha]_{\mathrm{D}}-33.8^{\circ}(c 0.51, \mathrm{MeOH})$; IR $v_{\max } / \mathrm{cm}^{-1} 1460,1413,1375,1267 ;{ }^{1} \mathrm{H}$ NMR $\left(\mathrm{CDCl}_{3}\right) \delta 0.94(6 \mathrm{H}, \mathrm{m}), 1.25-1.68(8 \mathrm{H}, \mathrm{m}), 1.32$ $(3 \mathrm{H}, \mathrm{s}), 1.49(3 \mathrm{H}, \mathrm{s}), 3.31(3 \mathrm{H}, \mathrm{s}), 3.47(1 \mathrm{H}, \mathrm{s}), 3.76(1 \mathrm{H}, \mathrm{d}, J 3.2 \mathrm{~Hz}), 4.55(1 \mathrm{H}, \mathrm{d}, J 5.8 \mathrm{~Hz})$, $4.85\left(1 \mathrm{H}, \mathrm{dd}, J_{1} 3.2 \mathrm{~Hz}, J_{2} 5.8 \mathrm{~Hz}\right), 4.96(1 \mathrm{H}, \mathrm{s}) ;{ }^{13} \mathrm{C} \mathrm{NMR}\left(\mathrm{CDCl}_{3}\right) \delta 14.2,14.4,16.2,16.3$, 23.8, 25.4, 37.6, 38.6, 54.0, 74.6, 80.0, 80.4, 84.8, 105.9, 112.3; FAB-MS m/z $289\left(\mathrm{MH}^{+}\right)$. HRMS Calcd for $\mathrm{C}_{14} \mathrm{H}_{25} \mathrm{O}_{5}$ [(M-Me $)^{+}$]: 273.1702. Found: 273.1709 .

\section{Methyl 2,3-O-(1-methylethylidene)-5,5-di- $C$-phenyl- $\alpha$-L-lyxofuranoside (12).}

To the solution of lyxofuranose $9 \mathrm{c}(534 \mathrm{mg}, 1.6 \mathrm{mmol})$ in a mixture of methylene chloride (7 $\mathrm{mL})$ and toluene $(2 \mathrm{~mL})$ tetrabutylammonium bromide $(80 \mathrm{mg}, 0.25 \mathrm{mmol}), 20 \%$ solution of sodium hydroxide $(5 \mathrm{~mL})$ and dimethyl sulfate $(0.52 \mathrm{~mL}, 5.4 \mathrm{mmol})$ were added. The reaction was carried out in a two-phase mixture under phase-transfer conditions for $12 \mathrm{~h}$ at $\mathrm{rt}$. Layers were separated; the aqueous layer was diluted with additional water $(30 \mathrm{~mL})$ and extracted with methylene chloride $(3 \times 10 \mathrm{~mL})$. Combined organic extracts were dried over $\mathrm{Na}_{2} \mathrm{SO}_{4}$, filtered and evaporated under reduced pressure. A yellow oil was purified by column chromatography (gradient ethyl acetate-petroleum ether 1:20 to 1:5) to give 12 (506 mg, 89\%) as a yellow oil, which crystallized as a white solid; mp $94-98{ }^{\circ} \mathrm{C}$ (petroleum ether); $[\alpha]_{\mathrm{D}}-$ $35.7^{\circ}$ (c 0.33, MeOH); IR $v_{\max } / \mathrm{cm}^{-1} 1493,1450,1383,1272,1213 ;{ }^{1} \mathrm{H}$ NMR $\left(\mathrm{CDCl}_{3}\right) \delta 1.18$ $(3 \mathrm{H}, \mathrm{s}), 1.54(3 \mathrm{H}, \mathrm{s}), 3.35(3 \mathrm{H}, \mathrm{s}), 4.54(1 \mathrm{H}, \mathrm{d}, J 5.8 \mathrm{~Hz}), 4.59\left(1 \mathrm{H}, \mathrm{dd}, J_{1} 3.0 \mathrm{~Hz}, J_{2} 5.8 \mathrm{~Hz}\right)$, $4.93(1 \mathrm{H}, \mathrm{d}, J 3.0 \mathrm{~Hz}), 5.02(1 \mathrm{H}, \mathrm{s}), 5.15(1 \mathrm{H}, \mathrm{br} \mathrm{s}), 7.24(6 \mathrm{H}, \mathrm{m}), 7.54(4 \mathrm{H}, \mathrm{m}) ;{ }^{13} \mathrm{C} \mathrm{NMR}$ (DMSO- $\left.d_{6}\right) \delta 24.1,25.7,55.0,78.1,80.1,80.9,84.9,107.2,112.9,125.3,125.5,126.6,126.8$, 127.9, 128.1, 144.8, 145.4; FAB-MS $m / z 339$ [(MH- $\left.\left.\mathrm{H}_{2} \mathrm{O}\right)^{+}\right]$. Anal. Calcd for $\mathrm{C}_{21} \mathrm{H}_{24} \mathrm{O}_{5} \times 0.3$ $\mathrm{H}_{2} \mathrm{O}: \mathrm{C}, 69.71 ; \mathrm{H}, 6.85$. Found: $\mathrm{C}, 69.59 ; \mathrm{H}, 6.62$. 